\title{
Attending to an object's color entails attending to its location: Support for location-special views of visual attention
}

\author{
YEHOSHUA TSAL and DOMINIQUE LAMY \\ Tel Aviv University, Tel Aviv, Israel
}

\begin{abstract}
Van der Heijden, Kurvink, de Lange, de Leeuw, and van der Geest (1996) argued that the results supporting the location-special view obtained by Tsal and Lavie (1988) were due to uncontrollable shifts of fixation, rather than reflecting the properties of the attentional system. In the present study, we present an improved variation of the Tsal and Lavie (1988) paradigm and reassert our claim that location is a special dimension. Subjects were presented with circular arrays of six letters of different colors. Three of the letters were enclosed by (Experiment 1) or superimposed on (Experiments 2,3, and 4) different colored shapes. The subjects were instructed to report the (target) shape with a given color (e.g., report whether the red shape was a square, a circle, or a triangle) and then either freely report letters from the array (Experiments 1, 2, and 4) or identify a prespecified target letter (Experiment 3). In all four experiments, performance was substantially better for the letter that appeared in the location of the to-be-reported shape (location letter) than for the letter that shared its color (color letter). We conclude that attending to the stimulus color entails directing attention to its location.
\end{abstract}

The special status of stimulus location in visual selective attention has been extensively debated in recent years. As van der Heijden, Kurvink, de Lange, de Leeuw, and van der Geest (1996) have suggested, "a brief look at current theorizing can make this clear. There are two groups of theories: the 'position-not-special' ones and the 'positionspecial' ones" (p. 1224).

Over the years, there has been a wide range of findings that provided support for the unique role of location in visual selection (e.g., Cave \& Pashler, 1995; Hoffman \& Nelson, 1981; Kim \& Cave, 1995; Luck, Fan, \& Hillyard, 1993; Posner, Snyder, \& Davidson, 1980; Theeuwes, 1989; Tsal \& Lavie, 1988, 1993; Vecera, 1994). For example, Tsal and Lavie (1993, Experiment 4) presented a cuing display consisting of two peripheral disks, one black and one colored (either pink or blue), followed by a probe display containing a target letter. Subjects were told to respond to the target letter only if the colored disk was pink. Although the location of the disk was entirely task irrelevant, the probe was detected faster when it appeared in the location previously occupied by the colored disk than when it appeared in the location of the black disk.

In contrast to the above studies, a large number of findings have provided support for the notion that location is not different in principle from other selection dimensions

This research was supported by Grant $061071055 \mathrm{I}$ from the Israel Science Foundation. We are grateful to Kyle Cave, Lex van der Heijden, and two anonymous reviewers for their helpful comments on an earlier draft. Correspondence concerning this article should be addressed to Y. Tsal, Department of Psychology, Tel Aviv University, Ramat Aviv, Tel Aviv 69978, Israel (e-mail: jehoshua@freud.tau.ac.il). (e.g., Baylis \& Driver, 1992, 1993; Duncan, 1984; Harms \& Bundesen, 1983; Humphreys, 1981). For example, using the Eriksen flanker paradigm, Baylis and Driver (1992) reported that distant incompatible distractors grouped with the target by color or by good continuation interfered with target identification more than did closer incompatible distractors that were not otherwise grouped with the target.

Recently, a controversy arose concerning the results obtained under a particular paradigm introduced by Tsal and Lavie (1988) that supported the location-special view. The uniqueness of this paradigm was that it assessed the effects of attending to location when location was completely irrelevant to the task. Van der Heijden et al. (1996) showed that these results were due to uncontrollable fixation shifts and, as such, did not reflect the properties of the attentional system. The present study is an improvement on our original paradigm. It shows that even under proper fixation conditions, attempting to attend to any aspect of a stimulus entails attending to its location.

Tsal and Lavie (1988, Experiment 1) presented a circular array containing three red, three green, and three brown letters. The subjects were instructed to report first one letter of a given color and then any other letters they could identify. The letters reported additionally were more often letters adjacent to the first reported letter (location letters) than letters with the same color as the first reported letter (color letters). Tsal and Lavie (1988) concluded that the selective processing of a target specified by its color is accomplished by attending to this target's location. They noted that this experiment may have suffered from a methodological difficulty: "Since three of the letters 
were always relevant, subjects may have shifted fixation to any array position prior to stimulus onset, since any random position was highly likely to include a letter of the relevant color in that or in an adjacent position" (p.17). Thus, the results may have reflected sensory differences in visual processing rather than attentional effects. Tsal and Lavie (1988) minimized this problem in Experiments 2 and 3, but in these experiments, the target letter was specified by its shape, rather than by its color.

In a recent series of experiments, van der Heijden et al. (1996) demonstrated that improper fixation could indeed provide the best account for Tsal and Lavie's (1988) results (Experiment 1). They showed that when forced to fixate the center of the display, the additional letters subjects reported were more often color letters than location letters. An advantage for location letters was found only under low-contrast conditions when subjects were not forced to fixate the fixation point.

In the series of experiments presented below, we eliminated the fixation problem investigated by van der Heidjen et al. (1996). Subjects had to report the form of the only shape in a circular array that had a given color, and then report letters from the array. One of the letters had exactly the same color as the shape (color letter), and another letter occupied exactly the same location as the shape (location letter). The target shape was equally likely to appear in any location. The letters were all of different colors and were randomly presented in the various positions. Thus, there was no benefit in shifting fixation to any position or region of the circular array prior to stimulus onset. Moreover, in the last experiment, in order to further ensure that the subjects would not shift their eyes, we followed the methodology used by van der Heijden et al. to control for eye movements. We included a sign in the center of the display, the form of which instructed subjects whether to respond or withhold their responses. Following van der Heijden et al.'s reasoning, we assumed that the central item would require foveal processing, thus necessitating central eye fixation.

In addition to the fact that the present study was designed to overcome this fixation problem, it also included a number of improvements over Tsal and Lavie's (1988) paradigm. The original rationale was that the location superiority effect obtained when the target was specified by its color and its location was completely irrelevant to the task demonstrated that attending to location is a mandatory process. However, it is important to note that this paradigm also involved several biases that minimized the likelihood of observing location responses even if subjects did attend to the target's location. Such biases may account for the color superiority effect obtained by van der Heijden et al. (1996).

The first bias concerned the fact that the color letters were potential targets for first-letter report. For instance, since there were three red letters in the array, when subjects were instructed to first report a red letter, all three red letters were potential targets and were thus likely to have undergone some level of processing. As a result, re- porting additional red letters may have reflected effects of the instructions rather than the persisting effects of the activation of redness. In contrast, since location was task irrelevant, effects of location could not have stemmed from top-down effects of instructions and could only indicate that attending to an item entailed activating a spatial representation. In the present study, we eliminated this problem, since the color letter used to assess the effects of attending to color was not a potential item for first-target report.

The second problem with this paradigm was that it may not have been sufficiently sensitive for demonstrating location effects, because it was clearly dimensionally asymmetrical. Indeed, the letters included in the color category were exactly of the same color as the first reported letter, whereas letters included in the location category did not occupy precisely the same location as the first letter reported, but only positions adjacent to it. Thus, obtaining location responses under these conditions requires additional assumptions about the shape and size of the attentional spotlight. In the present study, we eliminated this bias, since the location letters used to assess the effects of attending to location occupied precisely the same location as the target item.

Note that the above improvements only facilitate the assessment of the persisting effects of attending to location. The paradigm remains strongly biased against location, since the subject has to attend to the color of the shape and the locations of the shapes and letters are totally irrelevant to the task. Still, the new results presented here support the location-special view and reinforce our claim that when attending to the color of a given item, subjects cannot avoid attending to its location.

\section{EXPERIMENT 1}

In the present experiment, the subjects were presented with a circular display of six different letters of different colors. Three of the letters were enclosed by three different colored shapes (see Figure 1). The subjects were instructed to report the shape of a given color (e.g., report whether the red shape was a square, a circle, or a triangle) and then as many letters as they could. One of the letters had the same color as the target shape and was defined as the color letter. The letter enclosed by the target shape was defined as the location letter. The critical comparison concerned the frequency of report of color letters and of location letters. Finding that subjects indeed tend to report location letters more frequently than color letters would demonstrate that attending to the color of a shape entails that its location, although irrelevant, is activated, and more so than its color, which is task relevant. Such a finding would strongly support the idea that location is a special dimension. As in the studies of Tsal and Lavie (1988) and Van der Heijden et al. (1996), the subjects initially attended to a given color and then freely reported any letters they could. Unlike these two former studies, the location letter-namely, the letter used to assess the 


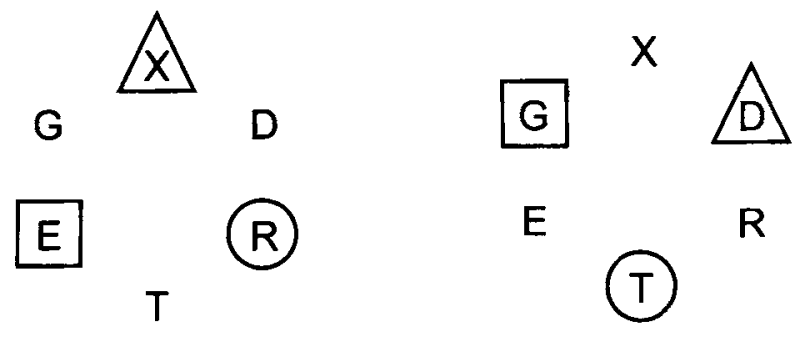

Figure 1. Examples of the stimuli used in Experiment 1.

effects of attending to location-occupied the same location as the shape with the target color, rather than a location adjacent to it.

\section{Method}

Subjects. The subjects were 8 undergraduates from Tel Aviv University. Three were paid subjects, and 5 participated as part of a course requirement. All had normal or corrected-to-normal vision.

Stimuli and Procedure. Stimulus presentation and data collection were controlled by an IBM PC/AT with a VGA graphic display. A chinrest was used to stabilize viewing distance at $43 \mathrm{~cm}$ so that $1 \mathrm{~cm}$ on the display corresponded to $1.33^{\circ}$ of visual angle. The subjects were presented with circular letter displays. Each display consisted of six different letters of different colors. The letters were randomly sampled from the entire alphabet. The colors were red (RGB values, 255/80/80; luminance, $21 \mathrm{~cd} / \mathrm{m})$, blue $(80 / 80 / 255$; $21 \mathrm{~cd} / \mathrm{m})$, green $(0 / 160 / 0 ; 32 \mathrm{~cd} / \mathrm{m})$, white $(255 / 255 / 255 ; 111 \mathrm{~cd} / \mathrm{m})$, magenta $(255 / 80 / 255 ; 37 \mathrm{~cd} / \mathrm{m})$, and yellow $(255 / 255 / 80 ; 98 \mathrm{~cd} / \mathrm{m})$. For each display, the letters, their colors, and their positions were randomly paired. Each letter subtended $1.33^{\circ}$ in height and $0.93^{\circ}$ in width, and the center-to-center interletter distance was $5.32^{\circ}$ of visual angle. The entire array subtended $9.31^{\circ}$ of visual angle in diameter and was centered on the fixation point. In each display, three of the letters were enclosed by three different colored shapes: a square ( $\left.\operatorname{side}=2.53^{\circ}\right)$, a circle $\left(\right.$ diameter $\left.=2.66^{\circ}\right)$, and an equilateral triangle ( side $=3.01^{\circ}$ ). The letter was centered inside the enclosing shape. One of these shapes was red, blue, or white (depending on block, as was specified below). The colors of the other two shapes were randomly sampled from the remaining five letter colors, with the constraint that a shape never enclosed a letter of the same color. The shapes were always separated by one intervening letter. In half of the displays, they occupied the top central position and the right and left bottom positions, and in the other half, they occupied the bottom center position and the right and left top positions. These two display types were randomly intermixed. Thus, each position was equally likely to contain the target shape, and there was therefore no gain in shifting fixation to any position prior to stimulus onset. Each stimulus was presented for $100 \mathrm{msec}$ and was immediately preceded by a gray central fixation cross presented for $1 \mathrm{sec}$. The subjects were instructed to report the shape (the target shape) of a given color (e.g., report whether the red shape was a square, a triangle, or a circle) and then as many letters as they could. The subjects were presented with three 45 -trial blocks, each preceded by 15 practice trials. Depending on block, the designated color for the target was red, blue, or white. The order of block presentation was randomized across subjects. The location letter was defined as the letter enclosed by the target shape. The color letter was defined as the letter sharing the color of the target shape. It was always separated from the location letter by one intervening letter and was always enclosed by a nontarget shape. All the other letters were defined as neutral. It was emphasized to the subjects before each block of trials that they had to report correctly any letters of the array, and as many as possible. Thus, the letters reported could be, but did not need to be, the letters sharing the target shape's color or location.

\section{Results and Discussion}

Trials in which the shape of the designated color was named incorrectly were excluded from analysis $(6 \%$ of the trials). Since the color letter and the location letter were enclosed by shapes, we analyzed the responses only for the neutral letter that was also enclosed by a shape, in order to make the three relevant response categories directly comparable. Thus, in each display there were one color letter, one location letter, and one relevant neutral letter. The latter served as a baseline for measuring any facilitation resulting from sharing the location or the color of the target shape. Table 1 shows the mean number of letters reported per trial in each category. On average, the subjects reported 1.29 relevant letters per trial (excluding the irrelevant neutral letters not enclosed by a shape). An overall analysis of variance (ANOVA) of letter category (location, color, or neutral) $\times$ color (red, blue, or white) $\times$ subjects revealed a highly significant effect for letter category $[F(2,14)=45.77, p<.0001]$. Further comparisons indicated that location letters were reported more frequently than color letters $[F(1,7)=37.51, p<.0005]$. This was true for each of the 8 subjects (see Table 2). The superiority of color letters over neutral letters was marginally significant $[F(1,7)=5.23, p=.056]$.

There was no significant effect of color $[F(2,14)=2.72]$, but there was a highly significant interaction effect between color and letter category $[F(4,28)=9.79, p>$ $.0001]$, indicating that the difference between reporting location letters and color letters was smaller when the target shape was white than when it was red or blue. However, additional comparisons showed that even for the white target shape, location letters were reported more frequently than color letters $[F(1,7)=7.07, p<.05]$ and color letters were reported more often than neutral letters $[F(1,7)=10.23, p<.05]$. The above interaction between color and letter category was also significant in all the subsequent experiments reported below. We will address this issue by presenting additional analyses in the General Discussion section.

The present findings provide strong support for the location-special view of visual attention. Not surprisingly, attending to the shape with the relevant color entailed that color letters were reported more often than neutral letters. More interestingly, it also entailed that location letters were reported more often than neutral letters and even more often than color letters.

Table 1

Mean Number of Letters Reported per Trial by Letter Category and Color Block in Experiment 1

\begin{tabular}{ccccc}
\hline Letter Category & Red & Blue & White & Mean \\
\hline Location & 0.75 & 0.81 & 0.59 & 0.72 \\
Color & 0.24 & 0.31 & 0.36 & 0.30 \\
Neutral & 0.30 & 0.26 & 0.25 & 0.27 \\
\hline
\end{tabular}


Table 2

Mean Number of Letters Reported per Trial by Subject and Letter Category in Experiment 1

\begin{tabular}{cccc}
\hline & \multicolumn{3}{c}{ Letter Category } \\
\cline { 2 - 4 } Subject & Location & Color & Neutral \\
\hline 1 & 0.38 & 0.09 & 0.03 \\
2 & 0.85 & 0.55 & 0.53 \\
3 & 0.72 & 0.00 & 0.01 \\
4 & 0.71 & 0.48 & 0.38 \\
5 & 0.85 & 0.43 & 0.46 \\
6 & 0.60 & 0.09 & 0.06 \\
7 & 0.83 & 0.62 & 0.54 \\
8 & 0.79 & 0.16 & 0.14 \\
\hline
\end{tabular}

\section{EXPERIMENT 2}

One might argue that in the first experiment, the letter and the surrounding shape formed a cohesive perceptual object (Treisman, Kahneman, \& Burkell, 1983). Hence, the superior performance for the location letters may have resulted from object-based processes, rather than reflecting the superiority of the location dimension. In order to eliminate this possibility, we disrupted the potential formation of a perceptual object in the second experiment by slightly displacing the shapes, so that they were superimposed on the letters rather than enclosing them. Note that in the first experiment, the spatial arrangement of the shape and the letter was not likely to disrupt performance. On the contrary, it enhanced the salience of the enclosed letter. In contrast, with the spatial arrangement prevailing in the next experiment, the subjects had to effortfully disentangle the shape from the letter, because the global percept of the potential object they formed would impair identification performance of both the shape and the letter.

\section{Method}

Subjects. The subjects were 9 undergraduates from Tel Aviv University. Five were paid subjects, and 4 participated as part of a course requirement. All had normal or corrected-to-normal vision.

Stimuli and Procedure. This experiment was exactly the same as the first one, with one exception. All the shapes were displaced to the right by $1.33^{\circ}$. As a result, the left side of the shape was superimposed on the letter, as is shown in Figure 2.

\section{Results and Discussion}

As in the first experiment, trials in which the shape of the designated color was named incorrectly were excluded from the analysis ( $7 \%$ of the trials). We analyzed responses only for the neutral letter that was superimposed on the shape, in order to make them comparable with the color and location responses. Table 3 shows the mean number of letters reported per trial in each category. On average, the subjects reported only 0.64 relevant letters per trial. This reduction in performance, relative to Experiment 1, can probably be attributed to the fact that the superimposition of the shapes and the letters made the task more difficult. Again, the ANOVA revealed a highly significant effect of letter category $[F(2,16)=24.81, p<$ $.0001]$. Further analyses indicated that, as in the first ex- periment, location letters were reported more frequently than color letters $[F(1,8)=26.28, p<.001]$. This was true for each of the 9 subjects (see Table 4). In contrast with the first experiment, however, there was no difference between the frequencies of reporting color letters and neutral letters $[F(1,8)=0.30]$. In order to account for the disappearance of the color letter effect, it is reasonable to assume that the increased difficulty of the task reduced the likelihood of reporting letters in addition to the location letter. As a result, floor effects may have masked the possible small difference between color letters and neutral letters. The ANOVA also revealed a significant main effect of color $[F(2,16)=6.9, p<.01]$. The interaction between color and letter category was significant $[F(4,32)=$ $32.11, p<.0001]$, indicating that the differences between reporting letters from the three categories were smaller for the white target shape than for the red and blue target shapes. Indeed, additional analyses carried out on responses to the white target shape revealed no significant differences between reporting letters from the three categories $[F(1,8)=1.69,1.96$, and 3.03 for the differences between location and color, color and neutral, and location and neutral letter categories, respectively]. Except for the white shape condition, which will be addressed in the General Discussion section, the results of the second experiment, in which the shapes and the letters were superimposed, show an even stronger location superiority effect than in Experiment 1, in which the shapes enclosed the letters. One may therefore conclude that the location superiority effect was mediated by space-based and not by object-based processes.

\section{EXPERIMENT 3}

The third experiment was designed to further contrast the relevant color dimension and the irrelevant location dimension under more constrained conditions. One might argue that in the first two experiments, subjects may, for some reason, have adopted a scanning strategy that biased selection in favor of the location letter, thereby diminishing the effect of the attentional priority of the color letter. In the present experiment, the free report task was replaced with the identification of a predesignated target letter. This method thus minimized strategic biases and provided a purer assessment of structural characteristics of the attentional system. We used the same displays as those in

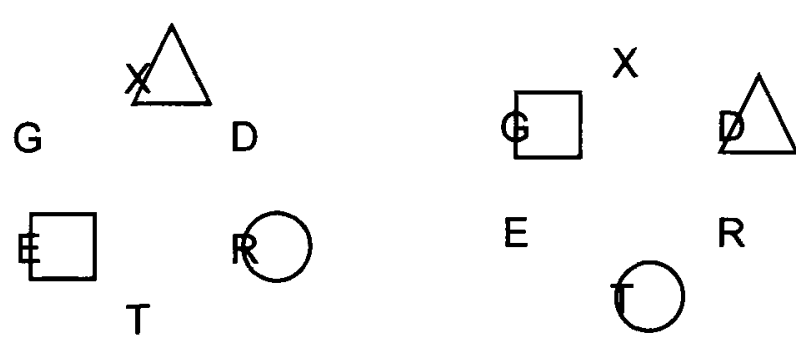

Figure 2. Examples of the stimuli used in Experiment 2. 
Table 3

Mean Number of Letters Reported per Trial by Letter Category and Color Block in Experiment 2

\begin{tabular}{lcccc}
\hline Letter Category & Red & Blue & White & Mean \\
\hline Location & 0.51 & 0.52 & 0.22 & 0.42 \\
Color & 0.09 & 0.09 & 0.17 & 0.12 \\
Neutral & 0.09 & 0.09 & 0.13 & 0.10 \\
\hline
\end{tabular}

Experiment 2. The subjects were required first to name the shape of a given color and then to report whether the display contained an $\mathrm{H}$ or a $\mathrm{U}$. This target letter was equally likely to appear in any position. We compared identification performance when the target letter was a location letter (i.e., appeared in the location of the target shape) with that when it was a color letter (i.e., shared the target shape's color). Superior performance for the location letters, relative to the color letters, would suggest that subjects initially attend to the location of the designated color and need to switch to the color dimension or shift to another location in order to report the color letter.

\section{Method}

Subjects. The subjects were 10 undergraduates from Tel Aviv University, who participated as part of a course requirement. Five subjects also participated in Experiment 2. All had normal or corrected-to-normal vision.

Stimuli and Procedure. The displays were identical to those in Experiment 2, except that each display contained a target letter, $\mathrm{H}$ in half of the displays and $U$ in the other half. This target letter was equally likely to appear in any position. The subjects were told first to report the shape of a given color and then to indicate which target letter (an $\mathrm{H}$ or a $\mathrm{U}$ ) they saw. In all other respects, the stimuli and procedure were identical to those in Experiment 2.

\section{Results and Discussion}

As in the first two experiments, we excluded trials in which the shape of the color was named incorrectly $(5 \%$ of the trials) and included only responses for neutral letters that were superimposed on a shape. Tables 5 and 6 show the proportions of correct target identification when the target letter was a location letter, a color letter, and a neutral letter.

The ANOVA indicated that the effect of letter category was significant $[F(2,18)=19.40, p<.0001]$. Further tests revealed that, as in Experiment 2, this effect was completely accounted for by the superiority of the location responses. The target was identified more often when it was a location letter than when it was a color letter $[F(1,9)=21.11, p<.005]$, and there was no difference between identifying color targets and neutral targets $[F(1,9)=$ 1.453]. The ANOVA revealed no significant effect of color $[F(2,18)=0.93]$, but there was a significant interaction between color and letter category $[F(4,36)=4.48$, $p<.005]$, indicating that the differences in identifying the target letter for the various categories were smaller for the white target letter than for the red and blue target letters. Indeed, additional comparisons between responses for the white target letters revealed no significant differences between reporting letters from the various cate- gories $[F(1,9)=.05,3.35$, and 2.95 for the differences between location and color letters, color and neutral letters, and location and neutral letters, respectively].

Because by eliminating free report, the present experiment minimized strategic factors, the most parsimonious explanation for the location superiority effect obtained here is that when the color of a given shape was attended to, the attentional spotlight was directed to its location. As a result, a location target appearing in a region receiving attentional priority was more likely to be picked up than was a target appearing in any other position outside the focus of attention.

\section{EXPERIMENT 4}

The present experiment was designed to further ensure that the location superiority effect was not contaminated by eye movements. Note again that, unlike in our previous study (Tsal \& Lavie, 1988), in the three experiments reported above, the subjects could not benefit from shifting their eyes to any peripheral position prior to stimulus onset, since each position was equally likely to include the target. Still, it may be possible that some subjects adopted an eye-shifting strategy in order to maximize performance on part of the trials. In order to eliminate this possibility, in the present experiment we adopted van der Heijden et al.'s (1996) method for minimizing eye movements by having subjects respond to a sign appearing in the center of the display and requiring foveal processing.

\section{Method}

Subjects. The subjects were 11 undergraduates from Tel Aviv University who participated as part of a course requirement. All had normal or corrected-to-normal vision.

Stimuli and Procedure. The stimuli and procedure were identical to those in Experiment 2, with two exceptions. First, the subjects were presented with 60 trials in each block. Second, a gray sign was now presented in the center of the display. It was either a plus sign ( $80 \%$ of the trials) or an X ( $20 \%$ of the trials). The subjects were instructed to respond only on those trials that included the plus sign and to withhold their responses on the remaining trials. This manipulation was identical to that used by van der Heijden et al. (1996). We followed their logic in assuming that the need to process the central symbol prior to response would prevent subjects from shifting attention to a peripheral position.

Table 4

Mean Number of Letters Reported per Trial by Subject and Letter Category in Experiment 2

\begin{tabular}{cccc}
\hline & \multicolumn{3}{c}{ Letter Category } \\
\cline { 2 - 3 } Subject & Location & Color & Neutral \\
\hline 1 & 0.53 & 0.09 & 0.12 \\
2 & 0.51 & 0.11 & 0.12 \\
3 & 0.16 & 0.12 & 0.07 \\
4 & 0.22 & 0.08 & 0.13 \\
5 & 0.63 & 0.20 & 0.14 \\
6 & 0.52 & 0.00 & 0.01 \\
7 & 0.38 & 0.14 & 0.10 \\
8 & 0.17 & 0.10 & 0.13 \\
9 & 0.56 & 0.18 & 0.14 \\
\hline
\end{tabular}


Table 5

Mean Proportions of Letters Reported per Trial by Letter Category and Color Block in Experiment 3

\begin{tabular}{lcccc}
\hline Letter Category & Red & Blue & White & Mean \\
\hline Location & .86 & .80 & .56 & .74 \\
Color & .37 & .34 & .57 & .43 \\
Neutral & .39 & .39 & .36 & .38 \\
\hline
\end{tabular}

\section{Results and Discussion}

We excluded trials in which the central target was responded to incorrectly (3\% misses and $6 \%$ false alarms) and in which the shape of the designated color was named incorrectly ( $4 \%$ of the trials). We analyzed only responses for the neutral letter that was superimposed on a shape to make these comparable with the color and location responses. The results are presented in Tables 7 and 8 . On the average, the subjects reported only 0.25 relevant letters per trial (excluding irrelevant neutral letters, not superimposed on a shape). This substantial reduction is obviously due to the need to process the central sign prior to responding to the peripheral target. Still, despite the very low level of correct letter reporting, a clear location superiority emerges from these tables. This observation was confirmed by an overall ANOVA, indicating that the effect of letter category was significant $[F(2,20)=22.25$, $p<.0001]$. Further tests revealed that, as in Experiment 2, this effect was completely accounted for by the superiority of the location responses. Location letters were reported more frequently than color letters $[F(1,10)=23.66$, $p<.001]$, and there was no difference between reporting color letters and neutral letters $[F(1,10)=0.05]$. Again, the main effect of color was not significant $[F(2,20)=$ $2.95]$, but this factor interacted with letter category $[F(4,40)=11.79, p<.0001]$, indicating that the superiority of location letters over color letters disappeared when the target was white. Indeed, for the white target shape, there was no difference between location letter and color letter reports $[F(1,10)=0.55]$, and the differences between reporting location letters and color letters relative to neutral letters were only marginally significant $[F(1,10)=3.83, p<.08$; and $F(1,10)=3.53, p<.09$, respectively]. The results of the present experiment provide an additional replication of the location superiority effect and indicate that this effect could not be due to an eye movement strategy.

\section{GENERAL DISCUSSION}

The present findings provide strong support for location-special views of visual attention. They show that when selecting a target on the basis of its color, subjects direct attention to its location.

In all four experiments, the subjects were instructed to name the shape of a given color and then report letters. The first experiment showed that the subjects most often reported the letter that was enclosed by the target shape, rather than the letter that had the target shape's color.
These results could not be attributed to the fact that the target shape and the enclosed location letter formed a perceptual object, since Experiment 2 replicated this result when the shape was superimposed on the letter rather than enclosing it. Under these conditions, the subjects had to segregate the superimposed letter and figure in order to process them independently. Any merging of the two into a single cohesive perceptual object would have interfered with the formation of response-relevant representations. Hence, the results could not reflect the operation of object-based processes but, rather, reflected that of space-based processes. Experiment 3 provided even stronger evidence for location superiority. It showed that under very constrained conditions, subjects could more easily detect a predesignated target when it occupied the location of the target letter than when it shared its color. This experiment eliminated free report and, therefore, minimized possible strategic effects. Hence, its results suggest that the location target appeared in a region receiving attentional priority and was therefore more likely to be picked up than was a target appearing in any other position outside the focus of attention. Experiment 4 eliminated the possibility that the subjects shifted their eyes to a peripheral position prior to display onset. In this experiment, the subjects were presented with a central sign and were required to identify it before responding. We followed the logic of van der Heijden et al. (1996) in assuming that the processing of this symbol would require foveal processing and thus necessitate fixation at the center of the display.

Another important finding is the minimal effects of the color of the target shape on performance. Although color specified the target and was thus the only task-relevant dimension, only in the first experiment did subjects report more color letters than neutral letters. This effect completely disappeared in all three subsequent experiments. This difference between Experiment 1 and the other experiments may be explained by the fact that the superimposition of the shapes and the letters made the task substantially more difficult in the latter experiments (as the reduction in overall performance in these experiments suggests), thus reducing the likelihood of reporting letters in addition to the location letter. This finding further em-

Table 6

Mean Proportions of Letters Reported per Trial by Subject and Letter Category in Experiment 3

\begin{tabular}{cccc}
\hline & \multicolumn{3}{c}{ Letter Category } \\
\cline { 2 - 4 } Subject & Location & Color & Neutral \\
\hline 1 & .92 & .62 & .47 \\
2 & .90 & .50 & .33 \\
3 & .40 & .50 & .50 \\
4 & .56 & .00 & .00 \\
5 & .87 & .68 & .62 \\
6 & .88 & .77 & .24 \\
7 & .92 & .52 & .61 \\
8 & .95 & .56 & .56 \\
9 & .54 & .04 & .04 \\
10 & .67 & .29 & .38 \\
\hline
\end{tabular}


Table 7

Mean Number of Letters Reported per Trial by Letter Category and Color Block in Experiment 4

\begin{tabular}{lcccc}
\hline Letter Category & Red & Blue & White & Mean \\
\hline Location & 0.19 & 0.24 & 0.08 & 0.17 \\
Color & 0.02 & 0.02 & 0.07 & 0.04 \\
Neutral & 0.02 & 0.02 & 0.04 & 0.04 \\
\hline
\end{tabular}

phasizes the superiority of location letters over color letters in showing that attending to the location of the target shape is a mandatory process.

A general problem involved in comparing the efficiency of selection by location versus that by color is the differential discriminability of values along these dimensions. In principle, one can never be sure that the particular values selected along each stimulus dimension are equally discriminable. For example, is the discrimination between red and blue as easy as the discrimination between locations separated by $2^{\circ}$ of visual angle? This problem is common to many studies that compare efficiency of selection on the basis of different dimensional cues (e.g., color and location). However, discriminability along a particular dimension may affect performance only if the task involves a judgment along this dimension. Because subjects had to respond to colors and because the locations these occupied in space were completely irrelevant to task demands, whether the particular positions containing the letters were more or less discriminable than the particular color values used in the present experiments was not likely to have any effect on performance.

Despite the arguments above, one can still argue that the location superiority demonstrated here may be contingent on the choice of the particular colors used in our experiments. Indeed, in all four experiments, the superiority of location letters over color letters was either reduced or eliminated when the target shape was white. White items were substantially brighter than red or blue items. It is therefore reasonable to assume that the color letter was reported more frequently in the white shape condition than in the red or blue shape conditions because it was white and thus more salient than because it shared the attended shape's color. This possibility is strongly supported by subsequent inspections of the data. They indicated that in all the experiments, the white letter was reported more often than the red and blue letters across letter categories. In order to eliminate the effects of differential sensory salience, we conducted additional analyses in all the experiments. We compared the relative report frequencies by letter category, separately for the white, red, and blue letters, so that the compared location, color, and neutral letters were all of the same color. The results are summarized in Table 9. As the table shows, in three of the four experiments (Experiments 1,2, and 4), location letters were reported more frequently than color letters for each of the three target shape colors, and this effect was highly significant. Moreover, the difference between reporting color letters and reporting neutral letters was sig- nificant only for the red and the blue target shapes in Experiment 1 . Hence, once the differential color salience was controlled for, the location superiority effect was obtained for all three colors of target shapes.

The above analyses make it possible to distinguish between bottom-up and top-down influences on letter reports. The results of all the experiments clearly show that the perceptual salience of letters had a considerable effect on performance (bottom-up influence), since the salient white letter was reported substantially more frequently than the red and the blue letters. The important point, however, is whether there was an interaction between color discriminability and top-down factors. That is, was location superiority overridden by top-down factors (task relevance of the color shared by the target shape and the color letter) when color discriminability was highest? That is, for white letters, were color letters reported more often than location letters? Inspection of the rightmost three columns of Table 9 clearly shows that the superiority of location letter reports over color letter reports was highly significant, even for the very discriminable white target. Note that in the last experiment, this superiority was even stronger for the white target than for the blue target. From this analysis, we may now conclude that when selecting the color of a given shape, the subjects attended to the location of this shape more than to its color, this effect being highly significant whether the color was highly or poorly discriminable. Therefore, although a direct comparison between the discriminability of location and color is not possible, the results above show that location superiority remains highly significant across substantial variations along the discriminability of the color dimension. Obviously, we would expect that despite this superiority, extreme conditions that impair location processing while facilitating color processing might override location superiority, but we would consider that to be the exception rather than the rule.

The evident discrepancy between the location superiority demonstrated in the present experiments and the color superiority obtained in the study of van der Heijden et al. (1996) can be explained best by the fact that the latter included unnecessary biases against location selection,

Table 8

Mean Number of Letters Reported per Trial by Subject and Letter Category in Experiment 4

\begin{tabular}{cccc}
\hline & \multicolumn{3}{c}{ Letter Category } \\
\cline { 2 - 4 } Subject & Location & Color & Neutral \\
\hline 1 & 0.03 & 0.01 & 0.02 \\
2 & 0.17 & 0.00 & 0.06 \\
3 & 0.25 & 0.08 & 0.04 \\
4 & 0.21 & 0.03 & 0.02 \\
5 & 0.28 & 0.01 & 0.01 \\
6 & 0.09 & 0.01 & 0.04 \\
7 & 0.83 & 0.62 & 0.54 \\
8 & 0.17 & 0.09 & 0.10 \\
9 & 0.01 & 0.02 & 0.01 \\
10 & 0.30 & 0.01 & 0.03 \\
11 & 0.15 & 0.02 & 0.03 \\
\hline
\end{tabular}


Table 9

Statistical Significance of the Differences

for Different Categories, Within Each Color

\begin{tabular}{|c|c|c|c|c|c|c|c|c|c|}
\hline \multirow[b]{2}{*}{ Experiment } & \multicolumn{3}{|c|}{ Location Versus Neutral } & \multicolumn{3}{|c|}{ Color Versus Neutral } & \multicolumn{3}{|c|}{ Location Versus Color } \\
\hline & Red & Blue & White & Red & Blue & White & Red & Blue & White \\
\hline 1 & ** & $* *$ & $* *$ & * & * & n.s. & ** & $* *$ & ** \\
\hline 2 & ** & ** & ** & n.s. & n.s. & n.s. & ** & ** & ** \\
\hline 3 & n.s. & * & $*$ & n.s. & n.s. & n.s. & n.s. & n. & n.s. \\
\hline 4 & * & $* *$ & ** & n.s. & n.s. & n.s. & * & $* *$ & ** \\
\hline
\end{tabular}

*approached significance $(p<.1) . \quad * *$ highly significant $(p<.01)$ n.s., nonsignificant.

first introduced by Tsal and Lavie (1988). The present results show that when the letter used to assess location responses occupies the same position as the target, rather than a position adjacent to it, and when responses for a single location item are compared with responses for a single color item, the location superiority effect is clearly demonstrated.

The present findings are inconsistent with locationnot-special views of visual attention (e.g., Broadbent, 1971; Bundesen, 1990; Duncan, 1981, 1984; Kahneman \& Henik, 1981; Neisser, 1967), which claim that selective processing is accomplished by continuously distinguishing between relevant and irrelevant information on the basis of the attribute of selection and that location is just one selection dimension, which may be most efficient but is not different in principle from other stimulus dimensions.

The present findings are in accord with location-special views of visual attention (e.g., Cave \& Wolfe, 1990; LaBerge \& Brown, 1989; Posner et al., 1980; Treisman, 1988, 1990; van der Heijden, 1992). For example, the present findings are easily accommodated by Treisman's feature integration theory. In this theory, attentional selection is essentially always accomplished via selection of locations in visual space as the attentional spotlight is directed at regions in the master map of locations. Furthermore, attention can be called to a particular location from a particular feature map. The target feature activates its link to the master map, thus enabling attention to focus on its location (Treisman, 1990).

Van der Heijden et al. (1996) contended that "the fact that nowadays position-special and position-not-special theories coexist peacefully already indicates that relevant decisive information is hard to come by. Either the evidence is simply irrelevant or the evidence is relevant but can be handled by both views when it is allowed to introduce additional assumptions" (p. 1224). We propose that the present study provides just that decisive evidence. This evidence supports the view that visual space constitutes a primary channel on the basis of which relevant information and irrelevant information are kept separate during the course of selective visual processing (Tsal, 1983; Tsal \& Lavie, 1988). The enhanced selective processing of items defined by color or by any other perceptual dimension is accomplished not by the opera- tion of internal structures representing these selection attributes, but by increasing the sensitivity of the locations the items occupy in space.

\section{REFERENCES}

BAYLis, G. C., \& Driver, J. (1992). Visual parsing and response competition: The effect of grouping factors. Perception \& Psychophysics, 51, 145-162.

Baylis, G. C., \& Driver, J. (1993). Visual attention and objects: Evidence for hierarchical coding of location. Journal of Experimental Psychology: Human Perception \& Performance, 19, 451-470.

Broadbent, D. E. (1971). Decision and Stress. London: Pergamon.

Bundesen, C. (1990). A theory of visual attention. Psychological Review, 97, 523-547.

CAVE, K. R., \& PASHLER, H. (1995). Visual selection mediated by location: Selecting successive visual objects. Perception \& Psychophysics, 57, 421-432.

CAVE, K. R., \& WolfE, J. M. (1990). Modeling the role of parallel processing in visual search. Cognitive Psychology, 22, 225-271.

DUNCAN, J. (1981). Directing attention in the visual field. Perception \& Psychophysics, 30, 90-93.

DUNCAN, J. (1984). Selective attention and the organization of visual information. Journal of Experimental Psychology: General, 113, 501-517.

Harms, L., \& BundesEn, C. (1983). Color segregation and selective attention in a nonsearch task. Perception \& Psychophysics, 33, 11-19.

Hoffman, J. E., \& Nelson, B. (1981). Spatial selectivity in visual search. Perception \& Psychophysics, 30, 283-290.

HumPhreYs, G. W. (1981). Flexibility of attention between stimulus dimensions. Perception \& Psychophysics, 30, 291-302.

Kahneman, D., \& Henik, A. (1981). Perceptual organization and attention. In M. Kubovy \& J. M. Pomerantz (Eds.), Perceptual organization (pp. 181-211). Hillsdale, NJ: Erlbaum.

KIM, M., \& CAVE, K. (1995). Spatial attention in visual-search for features and feature conjunctions. Psychological Science, 6, 376-380.

LABERGE, D., \& BRown, V. (1989). Theory of attentional operations in shape identification. Psychological Review, 96, 101-124.

LUCK, S. J., FAN, S., \& Hillyard, S. A. (1993). Attention-related modulation of sensory-evoked brain activity in a visual search task. Journal of Cognitive Neuroscience, 5, 188-195.

NeIsSER, U. (1967). Cognitive psychology. New York: Appleton-CenturyCrofts.

Posner, M. I., SNyder, C. R. R., \& Davidson, B. J. (1980). Attention and the detection of signals. Journal of Experimental Psychology: General, 109, 160-174.

THEEUWES, J. (1989). Effects of location and form cuing on the allocation of attention in the visual field. Acta Psychologica, 72, 177-192.

Treisman, A. M. (1988). Features and objects: The fourteenth Bartlett memorial lecture. Quarterly Journal of Experimental Psychology, 40A, 201-237.

Treisman, A. M. (1990). Variations on the theme of feature integration: A reply to Navon. Psychological Review, 97, 460-463.

Treisman, A. [M.], Kahneman, D., \& Burkell, J. (1983). Perceptual 
objects and the cost of filtering. Perception \& Psychophysics, 33, 527-532.

TSAL, Y. (1983). On interpreting the effects of location preknowledge: A critique of Duncan. Perception \& Psychophysics, 34, 297-298.

TSAL, Y., \& Lavie, N. (1988). Attending to color and shape: The special role of location in selective visual processing. Perception \& Psychophysics, 44, 15-21.

TSAL, Y., \& LAVIE, N. (1993). Location dominance in attending to color and shape. Journal of Experimental Psychology: Human Perception \& Performance, 19, 131-139.

van der HeIJden, A. H. C. (1992). Selective attention in vision. London: Routledge. yan der Hejjeen, A. H. C., Kurvink, A. G., de Lange, L., de Leeuw, F., \& VAN DER GEEST, J. N. (1996). Attending to color with proper fixation. Perception \& Psychophysics, 58, 1224-1237.

VeCERA, S. P. (1994). Grouped locations and object-based attention: Comment on Egly, Driver, \& Rafal (1994). Journal of Experimental Psychology: General, 123, 316-320.

(Manuscript received September 30, 1998; revision accepted for publication July 22,1999 .) 